\title{
Working memory in Cantonese and German speaking dyslexic children
}

\section{BACKGROUND}

The performance of visual and auditory working memory together with different automated central executive functions was investigated on the basis of four computerized, adaptive task sets with measurement of accuracy and reaction time.

\section{PARTICIPANTS AND PROCEDURE}

Eighty-six children selected from 192 dyslexic and nondyslexic children (mean age $=10.29$ years) in Hong Kong and Leipzig were matched on intelligence by using the Culture Fair test (CFT 20) and age. The used reading and writing tests were language specific but scientifically similar. Four task sets with visual material (dot and line patterns) and auditory material (tone sequences) were adapted and randomly presented by a computer. Mean and maximum accuracy and speed parameters were measured. The hypotheses of dyslexia deficits and Chinese superiority in working memory performance on nonverbal material were examined.

\section{RESULTS}

The Cantonese speaking children were found to have a working memory advantage in the speed measure on all four task sets with visual and auditory stimulus presentation, and in the accuracy measure on the auditory tasks only. Dyslexia deficits were only found in the Chinese sample for the maximum performance parameters and one auditory task set. In the German sample, the dyslexia deficits were found to be more generalized in the auditory matching and reproduction task sets concerning mean and maximum accuracy and speed parameters.

\section{CONCLUSIONS}

The novel approach in this study concerns the new paradigm of adaptive, time efficient testing of working memory functions with nonverbal, auditory and visual material.

\section{KEY WORDS}

visual and auditory working memory; central executive; logographic and alphabetic script 


\section{BACKGROUND}

During the last century, hundreds of scientists searched for the specific sources of dyslexia. One theory is related to deficits in different functions of working memory as a basis of reading and writing acquisition. An abundance of contradictory results leads to questions about the language-related, cultural, and subtype specificity of deficits. Prevalence rates of dyslexia varied from 3-5\% in Germany (Valtin, 1989) to $10 \%$ in Hong Kong Evelin Witruk (Chan, Ho, Tsang, Lee, \& Chung, 2007).

\section{WORKING MEMORY IN DYSLEXIA}

Impairment of working memory performance in dyslexic children has been found for visual and auditory stimuli with different paradigms and types of material, as Menghini, Finzi, Carlesimo, and Vicari (2011) stated.

\section{VISUAL WORKING MEMORY IN DYSLEXIA}

Regarding deficits in visual working memory, Ho and Bryant (1997) found deficits for Chinese poor readers in visual working memory tasks. So and Siegel (1997) have also reported that early visual memory skills are predictive of later reading performance in Chinese children. Ho and her colleagues $(2002,2004)$ also suggest that the major difficulties of Chinese dyslexic children lie in visual-orthographic processing and that some dyslexic children have difficulties in visual motion perception.

However, Vellutino (1987) denied a general deficit of the visual working memory. His dyslexic children were able to reproduce unknown Hebrew words and letters just as well as normal reading children. If the word list was in English, the dyslexic children performed significantly poorer than the control group, which refers to a deficit of dyslexics during storage and recall of linguistic information.

Compensation effects for deficits of visual working memory were found in a study by Witruk and Rosendahl (1999). For visual matching tasks and serial recall tasks, they found significant adaptations in the control group in a longitudinal and cross-sectional comparison of 7- and 9-year-old dyslexic children. For visual matching, a material-specific, non-general deficit in dyslexic children was found, and for the accuracy parameter, significantly higher error rates were observed with letters and dot patterns (Witruk, 1993, 1999; Witruk, Ho, \& Schuster, 2002).

\section{AUDITORY WORKING MEMORY IN DYSLEXIA}

The current discussion explores whether the reading and spelling difficulties of dyslexic children are based on auditory working memory deficits or on specific phonological working memory deficits with linguistic material. Some studies show that the dyslexia deficit is based in the auditory field in general and also involves phonology. For example, Lachmann (2007) found a lower Mismatch Negativity, which represents vast pre-attentional stimulus discrimination and memory comparisons in dyslexic children in comparison to nondyslexic children for linguistic stimuli and tone series. Auditory working memory deficits for nonlinguistic material were found by Helenius, Uutela, and Hari (1999), and for tone series by Hari and Renvall (2001).

Regarding the deficits of phonological working memory, research evidence has been more convergent. Ho, Law, and Ng (2000) and Ho and Lai (2000) were able to validate these phonological deficits in Chinese dyslexic children. In addition, phonological working memory deficits on sequential information such as in digit span tasks could be the root cause of some other deficits and are evident across child populations (Everatt et al., 2001).

Gathercole and Baddeley (1993) found delays of development regarding articulation speed, rehearsal of non-words and memory span for words in 8-, 11-, and 15-year-old dyslexic children. Phonological deficits were found in 8- and 11-year-old dyslexic children, but they were not found in 15-year-old dyslexic children.

\section{CENTRAL EXECUTIVE FUNCTIONS IN DYSLEXIA}

Proof of deficits in dyslexics in relation to central executive functions is found in only a few investigations. Schneider (2001) reported stronger activation of the frontal lobe in dyslexic children during mental rotation and sound connecting tasks. She interpreted these results as a stronger involvement of the central executive in dyslexic children on the basis of inefficient automation. The tasks used by Siegel and Ryan (1989a, b) involved executive functions during word recognition after sentence completion and counting. They found generalized working memory deficits in dyslexic children (age $=7-13$ years).

In summary, the results regarding the deficits of auditory and phonological working memory seem to be present with relatively high consistency. Deficits of visual working memory appear to depend strongly on the types of material used. The lower automatisation of central executive processes in dyslexics can be verified.

\section{DIFFERENCES BETWEEN CHINESE AND GERMAN LANGUAGE AND SCRIPT}

The present study compared working memory performance between Chinese and German dyslexic children. German is an alphabetic script with a medium degree of regularity compared to more regular 
alphabetic scripts. There are 78 graphemes (including 26 letters), each consisting of one, two or three letters, and 43 phonemes or phoneme combinations (Valtin, 1989; Nerius, 1987). The uniqueness of the German script is represented in the capitalization rules, composed words, and very long single words.

For Chinese orthography, the basic graphic unit is a character. Chinese is also described as a morphosyllabic system. Each Chinese character represents the smallest unit of meaning (i.e., morpheme), and characters are monosyllabic. There are many homophones at the character level. To avoid the problem of homophones, the majority of words are multisyllabic and about twothirds of them are bisyllabic (Taylor \& Taylor, 1995).

Word recognition in German is constrained on a sequential, one-dimensional order of signs like the letters of a word. Chinese word recognition requires visual discrimination in a two-dimensional field with finer differences among the signs in a word.

During the subsequent phonological phase, Chinese children have to learn the complex Chinese orthography-phonology correspondence (OPC) rules but in German the regularity of the grapheme-phoneme correspondence (GPC) rules helps to accomplish this phase rather quickly. Throughout the final orthographic phase, both Chinese and German children read words and word patterns automatically and have rapid access to their meaning.

\section{WORKING MEMORY FUNCTIONS IN READERS OF LOGOGRAPHIC AND ALPHABETIC SCRIPTS}

Empirical studies comparing working memory in users of logographic and alphabetic scripts have used different paradigms (same-different tasks, free recall, multiple choice tasks, Sternberg tasks, etc.) and different presentation modes with activation of visual and/or auditory working memory and linguistic/ nonlinguistic material (Sternberg, 1975).

Some studies used the natural units of working memory for the comprehension of oral and written language like words and word combinations and compared working memory performance of these language units in Chinese and European language. The pronunciation of Chinese characters depends on a one-to-one association between a distinct visual configuration and a syllable. Because of the high prevalence of homophones, the visual form rather than the pronunciation gives more disambiguating information concerning the identity of a character. This special characteristic of Chinese may explain the socalled visual superiority effect as a long-term modality effect (Liu, Zhu, \& Wu, 1992) and the superiority of visual short-term memory performance with lists of single words (Tzeng, 1982). According to Perfetti and Zhang (1995), Chinese readers articulated significantly more slowly and they used significantly more pauses, whereas German readers used a set pattern of pause positions. These findings stress the assumption that the unit of our short-term store and of the processing during reading aloud is the word and not the syllable.

Studies with linguistic and nonlinguistic materials give us a more general insight into the patterns of working memory performance of Chinese and Western speakers. Zheng (1998) demonstrated in a series of visually and auditorily presented working memory tasks in Chinese (Beijing, Weishan) and German (Munich) children that the Chinese superiority effect was connected with the use of Chinese linguistic material and is not general. He found a significant age effect and a significant advantage of the Chinese children in the forward and backward digit-span test from the Wechsler intelligence test. He explained the age differences in both countries with a cultural universality of working memory development. This means that memory competencies have similar developmental patterns in all cultures.

Luer, Becker, Lass, Fang, Chen, and Wang (1998) used digits, names of numbers, color squares and color names presented visually in two paradigms. The span task required active reproduction of a series of items. The Sternberg task which included a condition with a token system required searching for a target item in a set of items and reporting its presence or absence. They found significantly higher spans for all four kinds of material in Chinese students compared with German students. The Sternberg tasks included a condition with a token system and included the same four categories of visually presented materials and samples. The token condition had a significant effect only in the German sample. With tokens, the reaction time and the errors were lower compared to the condition without tokens in the German sample but not in the Chinese sample. Significant superiority in the Chinese students was found only for the condition without tokens in all four kinds of material. The Germans reached the level of reaction time and accuracy of the Chinese only with the token rewards. This suggests a higher motivation level in Chinese individuals in experimental situations. The present authors' interpretation is that there is no difference in the basics of working memory between Chinese and German speakers but the phonological loop of Chinese individuals can be packed with more and smaller chunks and the breaks between the chunks are shorter. This is an effect induced by using the Cantonese language.

\section{AIMS OF THE STUDY}

1. The working memory performance of Chinese and German normal reading children will be compared to that of dyslexic children. The hypothesized Chinese superiority effect will be tested specifically in elementary school children.
Cantonese and German dyslexic children 
2. The language dependency of working memory functions will be examined by comparison to nonlinguistic material.

3. The discrimination of dyslexics and normal reading children will be tested in Hong Kong and in Leipzig. The specificity of the dyslexia deficits should be noted in both regions.

\section{PARTICIPANTS AND PROCEDURE}

Evelin Witruk

\section{ASSESSMENT OF DEVELOPMENTAL DYSLEXIA IN HONG KONG AND LEIPZIG}

The diagnosis of dyslexia in both countries was based on the international classification systems ICD-10 (World Health Organization, 1993) and DSM-5 (American Psychiatric Association, 2013), and the Culture Fair Intelligence test (CFT 20; Weiss, 1987) was used in both countries. However, different reading and writing tests were used.

The sample of dyslexic children in Leipzig consisted of students in their second year in a special remedial class for dyslexics. They were diagnosed on the basis of a test battery by Weigt (1994) and the Zürich Reading Test. Diagnosis of children with dyslexia in Hong Kong was based on a standardized Chinese word-reading test, an essay-copying test, and two visual-orthographic tests. The assessments provide a dyslexia diagnosis with a high degree of reliability, validity and objectivity.

\section{SELECTION OF SAMPLES}

Four groups were matched on the basis of the following criteria:

1. Age matching was based on a criterion of mean value \pm 3 standard deviations $(S D)$.
2. IQ was matched for the four groups using the CFT 20 test (Weiss, 1987) with a criterion of mean value $\pm 2 S D$ with the requirement that all IQs be above 80 .

3. The grade was matched for the nondyslexic samples in both countries and for the dyslexic sample in Hong Kong. The grade could not be matched for the Leipzig dyslexic sample, because the dyslexics attended a special remedial class for two years and had to repeat the second grade.

These selection criteria led to a reduction of the whole sample from 192 participants in the original sample to 86 participants ( 57 male, 29 female) with an average age of 123.41 months $(S D=9.28$, range $=109-146$ months) and an average IQ of 103.70 $(S D=8.75$, range $=85-120)($ see Table 1$)$.

\section{MATERIAL}

In both countries, the same experimental procedure and the same materials were used. The following four developed task sets were used to examine several subsystems of working memory (see Figure 1).

In Set A, visual working memory together with highly automated demands on the central executive were investigated by using memory matching tasks involving visual dot patterns. The participant had to compare the two sequentially presented dot patterns and had to decide "same" or "different" by pressing one of two different computer keys.

In Set B, auditory working memory together with highly automated demands on the central executive were investigated using memory matching tasks of sequences of high $(780 \mathrm{~Hz})$, middle $(611 \mathrm{~Hz})$, and low pitches $(440 \mathrm{~Hz})$. The participant had to compare the two successively presented pure sine tone sequences and decide "same" or "different" by pressing one of

Table 1

Sample description

\begin{tabular}{|c|c|c|c|c|c|c|c|}
\hline \multirow{2}{*}{$\begin{array}{l}\text { Location } \\
\text { Sample } \\
\text { Variable }\end{array}$} & \multicolumn{2}{|c|}{ Hong Kong } & \multicolumn{2}{|c|}{ Leipzig } & \multicolumn{3}{|c|}{$F$-values } \\
\hline & $\begin{array}{c}\text { Non- } \\
\text { dyslexic }\end{array}$ & Dyslexic & $\begin{array}{c}\text { Non- } \\
\text { dyslexic }\end{array}$ & Dyslexic & $F$ & $F^{2}$ & $F^{3}$ \\
\hline No. of participants & 19 & 19 & 24 & 24 & - & - & - \\
\hline Male/Female & $14 / 5$ & $16 / 3$ & $11 / 13$ & $16 / 8$ & - & - & - \\
\hline Age (months) & $\begin{array}{c}122.68 \\
(11.13)\end{array}$ & $\begin{array}{c}122.58 \\
(11.35)\end{array}$ & $\begin{array}{r}123.75 \\
(10.24)\end{array}$ & $\begin{array}{l}124.63 \\
(3.62)\end{array}$ & 0.58 & 0.04 & 0.06 \\
\hline Grade & $\begin{array}{c}4.37 \\
(0.83)\end{array}$ & $\begin{array}{c}4.42 \\
(0.90)\end{array}$ & $\begin{array}{c}4.21 \\
(0.42)\end{array}$ & $\begin{array}{c}3.00 \\
(0.00)\end{array}$ & $35.05^{* * *}$ & $18.73^{* * *}$ & $22.29^{* * *}$ \\
\hline $\mathrm{IQ}$ & $\begin{array}{l}102.11 \\
(9.10)\end{array}$ & $\begin{array}{r}105.26 \\
(10.26)\end{array}$ & $\begin{array}{l}104.79 \\
(7.83)\end{array}$ & $\begin{array}{l}102.63 \\
(8.25)\end{array}$ & 0.00 & 0.07 & 1.94 \\
\hline
\end{tabular}

Note. $F^{1}-$ main effect "location", $F^{2}$ - main effect "sample", $F^{3}$ - interaction between "location and sample"; ${ }^{*} p<.05,{ }^{* *} p<.01,{ }^{* * *} p<.001$. 
A

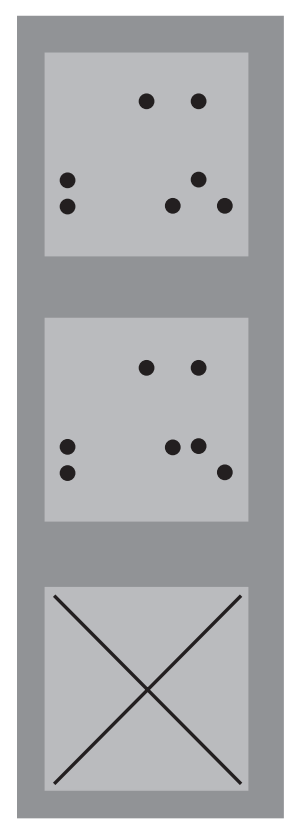

B

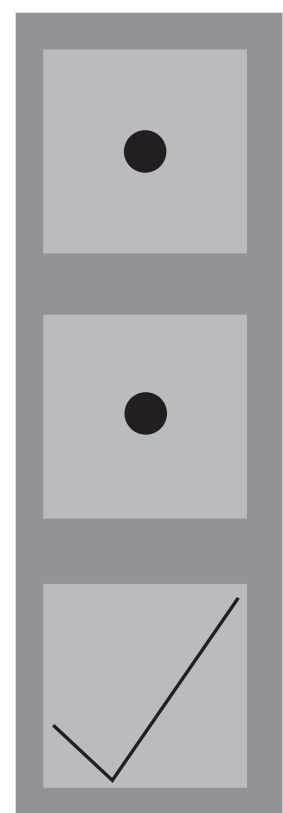

C

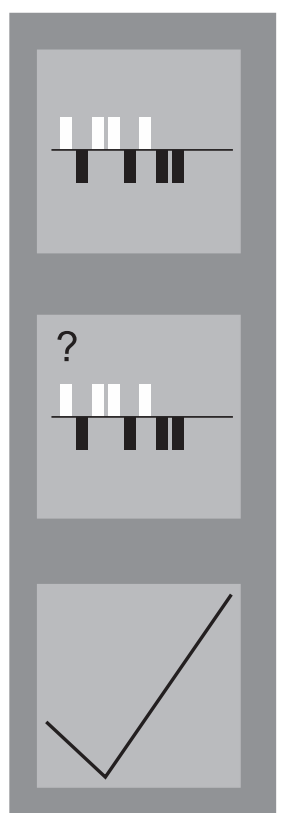

D

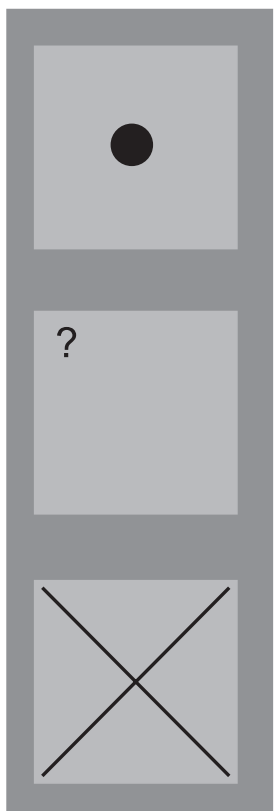

Cantonese and German dyslexic children

Figure 1. Examples of the stimulus material and feedback signs.

two different computer keys. The tone series grew progressively in number of tones across trials.

In Set C, the functions of visual working memory together with highly controlled demands on the central executive were investigated with visually presented line patterns. The participant had to reproduce the line patterns on the computer by pressing two different computer keys. The number of lines in a pattern grew progressively across trials.

In Set D, functions of auditory working memory together with highly controlled demands on the central executive were examined by presenting sequences of tones and requiring active reproduction of the tone sequences on the computer by pressing two different computer keys. The auditorily presented series of pure sine tones had an increasing number of tones with high $(780 \mathrm{~Hz})$ and low pitches $(344 \mathrm{~Hz})$.

Figure 1 shows examples of the stimulus materials for the memory matching tasks (A and B) and for the serial reproduction tasks $(\mathrm{C}$ and $\mathrm{D})$ and includes the feedback signs for right and wrong responses for the participant. These adaptive working memory tasks permit continuous adaptation to the degree of task difficulty by the participant. Visual and auditory matching and serial reproduction performance was scored for accuracy and latency.

\section{PROCEDURE}

The four task sets differed in presentation modality (visual or auditory) and in the degree of automation of executive function (same-different matching task or active serial reproduction task). Figure 2 shows the stimulus presentation times, the inter-stimulus intervals in the same-different matching tasks, the times for feedback, and breaks between the tasks. The times for decision and serial reproduction were open and were scored for latency in working memory performance. The order of the task sets was randomized, which allows for regulation of the individual level of difficulty of the working memory tasks. This prevents an overload of the child by starting with an easy task (two elements) and adding elements progressively until the child makes a mistake. Then the next task starts three steps back, and thus the individual limit of performance can be reached without overloading the child. Tasks based upon the same-different paradigm and the serial reproduction paradigms are presented both visually and auditorily. The items were given in random order following instructions and practice items. The presentation of each task set started with two elements in the form of points, lines or tones. A time limit of five minutes was set for each task set and feedback about the correctness of an answer was given.

\section{DESIGN}

A multivariate $2 \times 2 \times 2 \times 2$ experimental design had language (Cantonese, German) and reading ability (normal, dyslexic) as between-subject variables and type of task presentation (visually, auditorily) and type of working memory executive system (matching, active serial reproduction) as within-subject variables. 


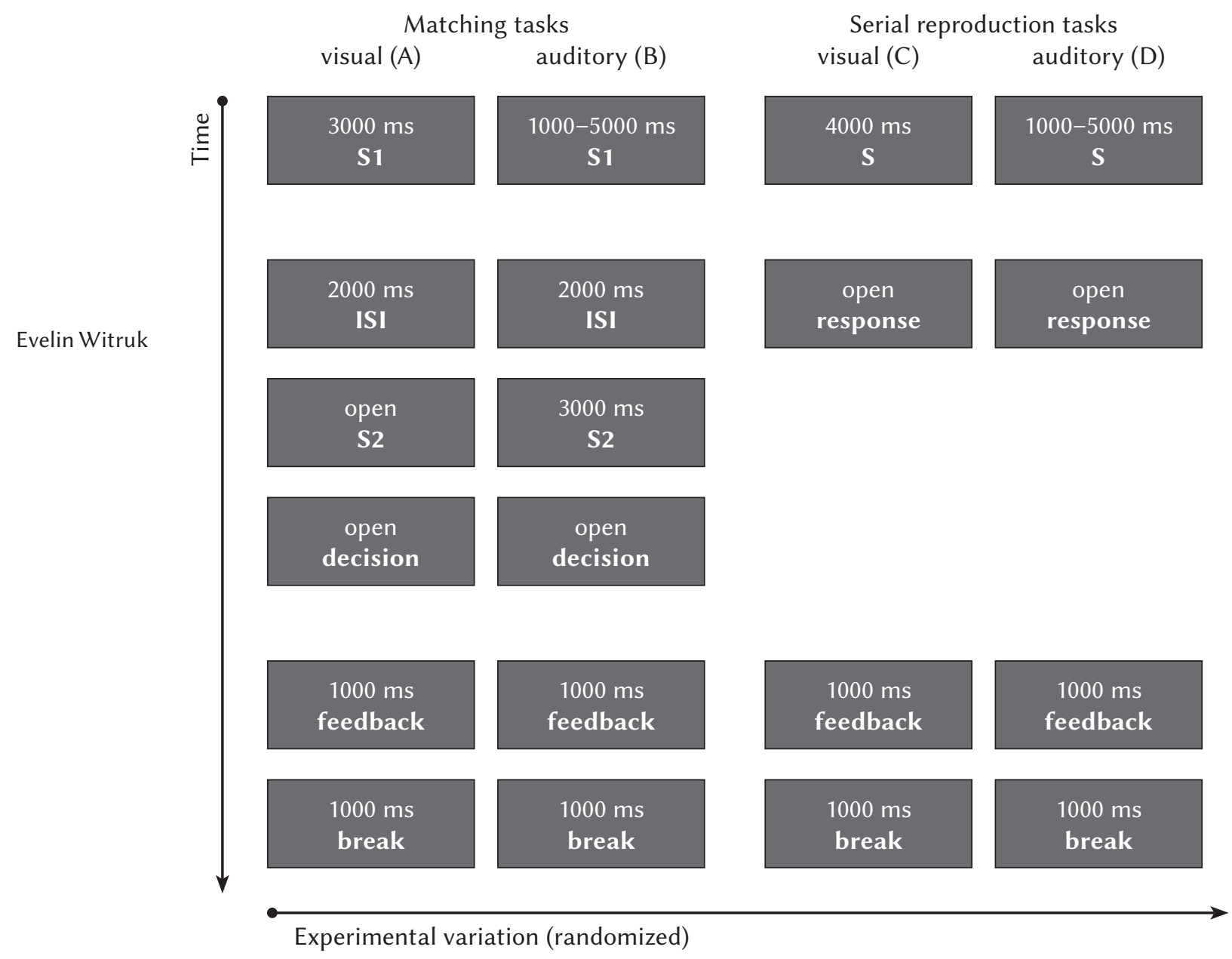

Note. S - stimulus presentation time, ISI - inter-stimulus interval

Figure 2. Experimental procedure.

Dependent variables were reaction time for correct responses and number of processed items as a latency working memory parameter. The accuracy parameter of working memory, the averaged level of accuracy, and the highest level of accuracy were measured.

\section{RELIABILITY AND VALIDITY \\ OF THE DEVELOPED EXPERIMENTAL MATERIAL}

The developed and implemented paradigm (Witruk, 1999) allows adaptive measurement of the performance of auditory and visual working memory. Table 2 shows the reliability of the developed experimental material as internal consistency of the material. The Chinese children have higher reliability than the Germans. One exception exists in the parameter "Number of proceed items", with very low internal consistency.

The retest-reliability after 6 months (measured only in the German samples) was on average 0.45 for the accuracy parameters and on average 0.41 for the temporal parameters. Accuracy and time parameters show higher stability for auditory tasks in comparison to visual tasks.

Regarding validity, we found an average correlation between intelligence, with accuracy measures of 0.30 and latency measures of 0.14 . The accuracy measures were more strongly linked to intelligence than the latency measures.

\section{RESULTS}

\section{STATISTICAL ANALYSES AND OUTLIER TREATMENT}

The data were analyzed with ANOVAs, $t$-tests, regression and correlation analyses for the performance measures of working memory. An $\alpha$ level of .05 was used for all statistical tests. The effect sizes in the current study have medium and high values in most of the cases (ANOVAs: higher than 0.25, $t$ - tests: higher than 0.50). We used the standardized effect size d' de- 
veloped by Bortz and Doering (2002) on the basis of Cohen (1988). The power value $(1-\beta)$ was developed on the basis of normal approximations of the $F$ and $t$ values (Faul \& Erdfelder, 1992) and it showed with one exception medium and high values over 0.50 .

The outlier treatment involved the dependent variables in the four task sets. Regarding the accuracy measure, we eliminated values exceeding $\pm 2.5 \times S D$. For the reaction time parameter, we eliminated values exceeding 2.5 seconds, because no strict task-related processes were to be expected in this range. Values below 0.25 seconds were also deleted because below this time level, no matching, decision, or reproduction processes were to be expected.

\section{LOGICAL FRAMEWORK OF CALCULATION AND RESULT PRESENTATION}

The structure of the result presentation follows an increasing aggregation process. It starts with the first section by comparing the performance parameters (mean and highest values) of the four task sets using ANOVAs with the interpretation of the main effects (location and sample) and the interactions. The second section is focused on the discrimination between dyslexic and nondyslexic children in both countries and directly represents the effect of reading level, and over its contrast, indirectly the effect of language. It discusses why the speed-accuracy relation in the different groups provides information about individual differences in the samples with reference to possible strategy differences in the processing of the matching and reproduction tasks with visual and auditory stimulus presentation.

\section{PERFORMANCE PARAMETERS IN THE FOUR ADAPTIVE WORKING MEMORY TASK SETS}

The main effects of the variables "location" and "sample" and their interaction were analyzed for accuracy and latency in the four task sets. Significant main effects of the variable "location" were found. ANOVAs showed significant advantages for Chinese children in the latencies of the working memory tasks. Chinese children showed significantly shorter reaction times in task set A, B, C and D. They processed a significantly higher number of items in task set $\mathrm{C}$. For the accuracy measures of the working memory tasks, we found advantages in the Chinese samples only in the task sets B and D, which are based on auditory presentation, and which are connected with the use of auditory working memory. Regarding the mean accuracy level, we found significantly higher values in the Chinese group in task set B and D. The highest accuracy level showed significant higher values in the Chinese group also in task sets B and D (see Table 3).
Table 2

Reliability of the developed experimental material

\begin{tabular}{lccc}
\hline Sample & Combined & Chinese & German \\
\hline \multicolumn{4}{c}{ Cronbach's $\alpha$} \\
\hline $\begin{array}{l}\text { Accuracy } \\
\text { parameters }\end{array}$ & .69 & .73 & .55 \\
$\quad$ Visual tasks & .63 & .74 & .47 \\
$\quad$ Auditory tasks & .76 & .82 & .72 \\
$\begin{array}{l}\text { Temporal } \\
\text { parameters }\end{array}$ & .57 & .62 & .52 \\
$\quad \begin{array}{l}\text { Averaged } \\
\text { reaction times }\end{array}$ & .78 & .74 & .74 \\
$\begin{array}{l}\text { Number of } \\
\text { proceed items }\end{array}$ & .37 & .51 & .31 \\
\hline
\end{tabular}

Cantonese and German dyslexic children
Significant main effects of the variable "sample" were found in task set B for the parameters highest level of accuracy and number of processed items, as well as in task set D for the parameters mean accuracy level and reaction time for correct responses. In these task sets, we found significant deficits of the dyslexic samples in Hong Kong and in Leipzig.

Significant interactions between the variables "location" and "sample" were found, particularly in task set D with auditory presentation and serial reproduction demand in the temporal working memory measure of reaction times for correct responses, and also in the averaged accuracy parameter, as well as in the highest level of accuracy. In addition, significant interactions were found in the number of processed items on task sets B and task set C. These significant interactions between the main factors describe a divergent pattern of performance of the dyslexic and nondyslexic samples in both countries. The results show a significant superiority effect of the Chinese children in the latency measure in all four task sets. Their advantages in accuracy are only significant in tasks with auditory stimulus presentation and demands on auditory working memory (see Table 3).

\section{DISCRIMINATION BETWEEN DYSLEXIC \\ AND NONDYSLEXIC CHILDREN IN HONG KONG AND IN LEIPZIG}

A statistical comparison between Chinese dyslexic and nondyslexic children is only possible in task set $\mathrm{B}$ with auditory stimulus presentation and matching demands and shows significance for the accuracy parameter "highest level of accuracy" $t(36)=2.32$, $p=.026$ with an effect size $d^{\prime}=0.75$ and a power value $(1-\beta)=1.00$ and for the latency measure "num- 
Table 3

Mean performance and maximum performance (cursive) in the four task sets (mean value and standard deviation in brackets)

\begin{tabular}{|c|c|c|c|c|c|c|c|}
\hline \multirow{2}{*}{$\begin{array}{l}\text { Location } \\
\text { Samples } \\
\text { parameters }\end{array}$} & \multicolumn{2}{|c|}{ Hong Kong } & \multicolumn{2}{|c|}{ Leipzig } & \multicolumn{3}{|c|}{$F$-values } \\
\hline & $\begin{array}{c}\text { Non- } \\
\text { dyslexic }\end{array}$ & Dyslexic & $\begin{array}{c}\text { Non- } \\
\text { dyslexic }\end{array}$ & Dyslexic & $F^{1}$ & $F^{2}$ & $F^{3}$ \\
\hline 1 & $8.09(2.78)$ & $8.50(2.36)$ & $8.26(2.26)$ & $8.83(2.72)$ & 0.21 & 0.80 & 0.02 \\
\hline 2 & $7.63(2.56)$ & $7.48(1.74)$ & $6.60(2.41)$ & $5.27(1.53)$ & $12.72^{* * *}$ & 2.64 & 1.67 \\
\hline 3 & $6.11(1.80)$ & $6.16(0.87)$ & $6.53(1.31)$ & $6.65(1.13)$ & 2.57 & 0.09 & 0.02 \\
\hline 4 & $5.03(0.87)$ & $5.23(0.89)$ & $5.24(0.82)$ & $4.23(0.88)$ & $4.51^{*}$ & $4.77^{*}$ & $10.71^{* *}$ \\
\hline 5 & $13.58(2.65)$ & $14.21(3.31)$ & $13.29(3.12)$ & $13.29(3.06)$ & 0.83 & 0.23 & 0.23 \\
\hline 6 & $11.79(2.68)$ & $10.16(1.50)$ & $9.67(2.46)$ & $9.00(2.40)$ & $10.61^{* *}$ & $5.21^{*}$ & 0.92 \\
\hline 7 & $9.05(1.78)$ & $9.21(1.23)$ & $9.21(1.50)$ & $9.21(1.22)$ & 0.06 & 0.06 & 0.06 \\
\hline 8 & $7.53(1.07)$ & $7.95(0.97)$ & $7.83(0.92)$ & $6.71(1.00)$ & $4.72^{*}$ & 2.69 & $13.00^{* * *}$ \\
\hline 9 & $1.51(0.27)$ & $1.51(0.33)$ & $1.51(0.27)$ & $1.67(0.46)$ & $8.56^{* *}$ & 0.59 & 0.55 \\
\hline 10 & $0.71(0.21)$ & $0.65(0.25)$ & $0.75(0.21)$ & $0.93(0.47)$ & $5.50^{*}$ & 0.70 & 3.06 \\
\hline 11 & $0.89(0.23)$ & $0.88(0.19)$ & $0.98(0.19)$ & $1.06(0.24)$ & $9.11^{* *}$ & 0.57 & 0.95 \\
\hline 12 & $0.89(0.15)$ & $0.90(0.14)$ & $0.97(0.14)$ & $1.19(0.29)$ & $16.65^{* * *}$ & $6.74^{*}$ & $6.49^{*}$ \\
\hline 13 & $34.47(1.22)$ & 34.37 (1.38) & $33.96(1.76)$ & $34.21(4.40)$ & 0.34 & 0.02 & 0.10 \\
\hline 14 & $23.47(0.51)$ & $22.95(0.52)$ & $24.54(1.69)$ & $22.46(2.25)$ & 0.76 & $15.45^{* * *}$ & $5.50^{*}$ \\
\hline 15 & $27.95(2.34)$ & $28.00(2.13)$ & $27.29(2.96)$ & $25.13(2.51)$ & $10.29^{* *}$ & 3.69 & $4.07^{*}$ \\
\hline 16 & $36.79(3.54)$ & 37.11 (4.08) & $36.54(3.16)$ & $36.38(6.58)$ & 0.24 & 0.01 & 0.06 \\
\hline
\end{tabular}

Note. $F^{1}$ - main effect "location", $F^{2}$ - main effect "sample", $F^{3}$ - interaction between "location and sample", the mean accuracy parameter (1-4), the highest accuracy value (5-8), the mean reaction times for correct responses (9-12), the total number of processed items $(13-16) .{ }^{*} p<.05,{ }^{* *} p<.01,{ }^{* * *} p<.001$.

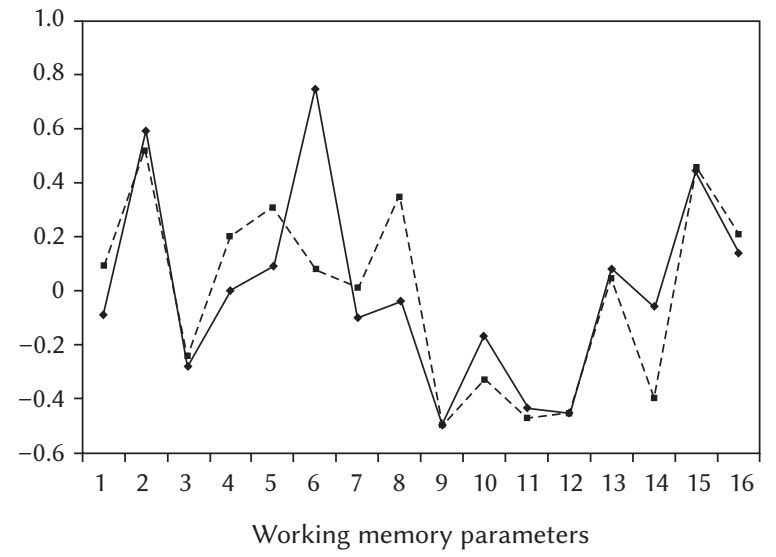

$\rightarrow$ Chinese Nondislexics $\quad--$ Chinese Dislexics

Note. $F^{1}$ - main effect "location", $F^{2}$ - main effect "sample", $F^{3}-$ interaction between "location and sample", the mean accuracy parameter (1-4), the highest accuracy value (5-8), the mean reaction times for correct responses (9-12), the total number of processed items (13-16) during sets A-D (transformed into Z-values). ${ }^{*} p<.05,{ }^{* *} p<.01,{ }^{* *} p<.001$.

Figure 3. Working memory performance profiles in Chinese dyslexic and nondyslexic children.

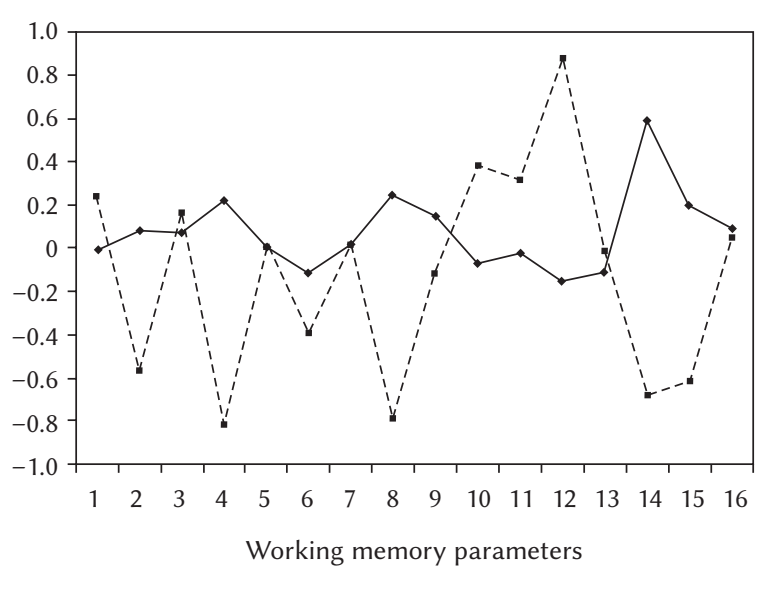

$\rightarrow$ German Nondislexics $\quad$-- German Dislexics

Note. $F^{1}$ - main effect "location", $F^{2}$ - main effect "sample", $F^{3}$ interaction between "location and sample", the mean accuracy parameter (1-4), the highest accuracy value (5-8), the mean reaction times for correct responses (9-12), the total number of processed items (13-16) during sets A-D (transformed into Z-values). ${ }^{*} p<.05,{ }^{* *} p<.01,{ }^{* * *} p<.001$.

Figure 4. Working memory performance profiles in German dyslexic and nondyslexic children. 
ber of processed items" $t=3.13, p=.003, d^{\prime}=1.02$, $(1-\beta)=1.00$. This finding explains the phonological deficit of Chinese children reported by Ho and Bryant (1997). In all other parameters and task sets, there were no significant differences between dyslexic and nondyslexic children in Hong Kong. That means the adaptive task series with the time limitation of five minutes were relatively easy for the Chinese children. They were not brought to their performance limits.

Between the German dyslexic and nondyslexic samples, we found significant differences in the sense of dyslexia deficits in the auditory task set B for the mean accuracy parameter, $t=2.28, p=.027$, $d^{\prime}=0.23,(1-\beta)=0.28$ and the number of processed items, $t=3.63, p=.001, d^{\prime}=1.05,(1-\beta)=1.00$. In the auditory task set $\mathrm{D}$ the dyslexic group showed significantly lower mean accuracy values than the nondyslexic group, $t=4.17, p<.001, d^{\prime}=1.20$, $(1-\beta)=1.00$, lower highest accuracy level, $t=4.06$, $p<.001, d^{\prime}=1.17,(1-\beta)=1.00$, and longer reaction times, $t=-3.30, p=.002, d^{\prime}=0.96,(1-\beta)=1.00$. The German dyslexics differed very strongly from the nondyslexics during auditory stimulus presentation and activation of auditory working memory (see Table 3). In task set $C$ the German dyslexics showed a significantly lower number of processed items than the nondyslexic group, $t=2.74, p=.009, d^{\prime}=0.79$, $(1-\beta)=0.98$.

\section{DISCUSSION}

The present findings demonstrate the Chinese superiority effect for working memory achievement for elementary school children. Their superiority was shown in the latency measures in all four task sets and in the accuracy measures in the auditory task sets.

The present findings support the strong impact of language systems on working memory performance, which was measured on nonverbal material (Smythe, Everatt, Gyarmathy, Ho, \& Groeger, 2003). The Chinese language system makes it necessary for oral communication to modulate the pitches of syllables and to rely on context. Therefore, learning the Chinese language is accompanied with a "natural" and highly efficient training of working memory and no deficits of dyslexia were discernible in the investigated areas. Our results can be also interpreted as an instance of the Chinese superiority effect. The finding of Liu et al. (1992) shows that lexical access for Chinese logographs is more direct and faster than for alphabetic words because the logographs are more unique in shape or more discriminated than alphabetic words. Our findings are also consistent with the results of Stevenson et al. (1986) and Luer et al. (1998), who found significant advantages for Chinese children and adults on visually presented, verbal working memory tasks.
The German language system, with its multisyllabic word constructions and combinations (except homophones), reaches an unambiguous meaning at the level of the word. Understanding spoken German requires less use of context and thus less maintenance of short units. The significant inferiority in performance of German children in the auditory task sets for accuracy and speed can be interpreted as a result of weaker "natural" training. It results in extremely visible significant deficits of performance for dyslexic children mostly related to auditory presentation of stimuli and combined with a demand for low controlled matching processing and high controlled reproduction processing.

The findings lead us to the question of whether in addition to the language differences we have to consider cultural differences as well. The better memory performance of Chinese children is probably due to the drilling practice in the educational system in Hong Kong emphasizing memorization and speed. Therefore, the cultural differences (in terms of educational practices) together with the language differences may contribute to the performance differences in the two regions.

Chinese dyslexic and nondyslexic children were discriminated by maximum performance measure and auditory task set B exclusively. This finding supports the phonological and the automatization deficits of Chinese dyslexic children also reported by Ho, Law, and Ng (2000). The discrimination between German dyslexic and nondyslexic children appears in auditory task sets B and D for both mean and maximum performance measures. This finding is relevant for theories of a phonological core deficit of dyslexics (Tallal, Galaburda, Llinas, \& von Euler, 1993; Share, 1994).

Regarding the impact of the dyslexia deficits on the modality-specific working memory systems and the central executive functions, there was no difference between dyslexic and nondyslexic children in Hong Kong. The highest discrimination between German dyslexic and nondyslexic children can be seen in auditory and serial reproduction tasks. This means, in view of the working memory model of Cowan (1995), that dyslexic children who are learning a medium regular script such as German have the main deficit in the auditory working memory and in higher controlled central executive functions.

Further research should clarify the dyslexia subtype assumption for dyslexics from different language/cultural environments by expansion of the samples. An increase in the difficulty of our working memory tasks realized by an extension of the adaptive procedure should lead to better discrimination between the Chinese dyslexic and nondyslexic children. In further studies, we may include dyslexic children, adolescents, and adults for examination of the age-compensation hypothesis of working mem-
Cantonese and German dyslexic children 
ory functions, discussed by Witruk $(2004,2005)$. The interdependence between early elementary auditory, visual perception performance, and later highly complex processes of working memory should also be investigated in the framework of different language and script systems as it is still an unsettled question in experimental dyslexia research.

\section{ACKNOWLEDGEMENTS}

Evelin Witruk

This investigation was supported by the German Academic Exchange Service (DAAD) and the Hong Kong Research Council. We are grateful for the help of Prof. Dr. Connie Suk-Han Ho from the University of Hong Kong, and the scientific assistants Dr. Yumi Lee, Dr. Shally Novita, and Dipl.-Psych. Thérèse Thuemler.

\section{RefERENCES}

American Psychiatric Association (2013). Diagnostic and Statistical Manual of Mental Disorders. DSM-5 ( $5^{\text {th }}$ ed.). Washington, DC: American Psychiatric Association.

Bortz, J., \& Doering, N. (2002). Forschungsmethoden und Evaluation fuer Human- und Sozialwissenschaftler ( $3^{\text {rd }}$ ed.) [Research methods and evaluation for human and social scientists ( $3^{\text {rd }}$ ed.)]. Berlin: Springer.

Chan, D. W., Ho, C. S. H., Tsang, S. M., Lee, S. H., \& Chung, K. K. H. (2007). Prevalence, gender ratio, and gender differences in reading-related cognitive abilities among Chinese children with dyslexia in Hong Kong. Educational Studies, 33, 249-265. https://doi.org/10.1080/03055690601068535

Cohen, J. (1988). Statistical power analysis for the behavioral sciences $\left(2^{\text {nd }}\right.$ ed.). Hillsdale, NJ: Lawrence Erlbaum Associates.

Cowan, N. (1995). Attention and memory. An integrated framework. New York: Clarendon.

Everatt, J., Groeger, J., Smythe, I., Baalam, S., Richardson, J., \& McNamara, S. (2001). Dyslexia and deficits in short-term memory: phonological versus sequential explanations. Abstract on $5^{\text {th }} B D A$ International Conference. York, UK.

Faul, F., \& Erdfelder, E. (1992). GPOWER: A priori-, post hoc-, and compromise power analyses for $M S$ DOS [computer program]. Bonn: Bonn University.

Gathercole, S. E., \& Baddeley, A. D. (1993). Working memory and language. East Sussex, UK: Lawrence Erlbaum Associates.

Hari, R., \& Renvall, H. (2001). Impaired processing of rapid stimulus sequences in dyslexia. Trends in Cognitive Sciences, 5, 525-532. https://doi.org/10.1016/ S1364-6613(00)01801-5

Helenius, P., Uutela, K., \& Hari, R. (1999). Auditory stream segregation in dyslexic adults. Brain, 122, 907-913. https://doi.org/10.1093/brain/122.5.907
Ho, C. S. H., \& Bryant, P. (1997). Phonological skills are important in learning to read Chinese. Developmental Psychology, 33, 946-951. https://doi. org/10.1037/0012-1649.33.6.946

Ho, C. S. H., Chan, D. W. O., Tsang, S. M., \& Lee, S. (2002). The cognitive profile and multiple-deficit hypothesis in Chinese developmental dyslexia. Developmental Psychology, 38, 543-553. https:// doi.org/10.1037//0012-1649.38.4.543

Ho, C. S. H., Chan, D., Tsang, S. M., Lee, S. H., \& Luan, V. H. (2004). Cognitive profiling and preliminary subtyping in Chinese developmental dyslexia. Cognition, 91, 43-75. https://doi.org/10.1016/ S0010-0277(03)00163-X

Ho, C. S. H., \& Lai, D. N. C. (2000). Naming-speed deficits and phonological memory deficits in Chinese developmental dyslexia. Learning and Individual Differences, 11, 173-186. https://doi.org/10.1016/ S1041-6080(00)80004-7

Ho, C. S. H., Law, T. P. S., \& Ng, P. M. (2000). The phonological deficit hypothesis in Chinese developmental dyslexia. Reading and Writing: An Interdisciplinary Journal, 13, 57-79. https://doi.org/ 10.1023/A:1008040922662

Lachmann, T. (2007). Basic determinants of specific learning disabilities in reading: Experimental exploration of component functions in reading and of deficits in their coordination. Kaiserslautern: Verlag der Universität Kaiserslautern.

Liu, I., Zhu, Y., \& Wu, J. (1992). The long-term modality effect: In search of differences in processing logographs and alphabetic words. Cognition, 43, 3166. https://doi.org/10.1016/0010-0277(92)90031-C

Luer, G., Becker, D., Lass, U., Fang, Y., Chen, G., \& Wang, Z. (1998). Memory span in German and Chinese: Evidence for the phonological loop. European Psychologist, 3, 102-112. https://doi.org/10. 1027//1016-9040.3.2.102

Menghini, D., Finzi, A., Carlesimo, G. A., \& Vicari, S. (2011). Working memory impairment in children with developmental dyslexia: Is it just a phonological deficity? Developmental Neuropsychology, 36, 199-213. https://doi.org/10.1080/87565641.2010. 549868

Nerius, D. (1987). Deutsche Orthographie [German orthography]. Leipzig: VEB Bibliographisches Institut.

Perfetti, C. A., \& Zhang, S. (1995). Very early phonological activation in Chinese reading. Journal of Experimental Psychology. Learning, Memory and Cognition, 21, 24-33. https://doi.org/10.1037/02787393.21.1.24

Schneider, A. (2001). EEG-coherence-analysis for examining a general automatizational deficit in dyslexic children. Abstract on $5^{\text {th }}$ BDA International Conference. York, UK.

Share, D. L. (1994). Deficient phonological processing in disabled readers implicates processing defi- 
cits beyond the phonological module. In K. P. van den Bos, L. S. Siegel, D. J. Bakker, \& D. L. Share (Eds.). Current directions in dyslexia research (pp. 149-167). Lisse: Swets \& Zeitlinger.

Siegel, L. S., \& Ryan, E. B. (1989a). The development of working memory in normally achieving and subtypes of learning disabled children. Child Development, 60, 973-980. https://doi.org/10.2307/1131037

Siegel, L. S., \& Ryan, E. B. (1989b). Subtypes of developmental dyslexia: The influence of definition variables. Reading and Writing: An Interdisciplinary Journal, 1, 257-287. https://doi.org/10.1007/ BF00377646

Smythe, I., Everatt, J., Gyarmathy, É., Ho, C. S. H., \& Groeger, J. A. (2003). Short-term memory and literacy: A cross-language comparison. Educational and Child Psychology, 20, 37-50.

So, D., \& Siegel, L. S. (1997). Learning to read Chinese: Semantic, syntactic, phonological and working memory skills in normally achieving and poor Chinese readers. Reading and Writing: An Interdisciplinary Journal, 9, 1-21. https://doi.org/10.1023/A:10079 63513853

Sternberg, S. (1975). Memory scanning. New findings and current controversies. Quarterly Journal of Experimental Psychology, 27, 1-32. https://doi.org/10. 1080/14640747508400459

Stevenson, H. W., Stigler, J. W., Lee, S., Lucker, G. W., Kitamura, S., \& Hsu, C. (1986). Cognitive performance and academic achievement of Japanese, Chinese, and American children. In S. Chess \& A. Thomas (Eds.), Annual progress in child psychiatry and child development (pp. 324-350). Philadelphia, PA: Brunner \& Mazel.

Tallal, P., Galaburda, A. M., Llinas, R. R., \& von Euler, C. (Eds.). (1993). Temporal information processing in the nervous system: Special reference to dyslexia and dysphasia. Annals of the New York Academy of Sciences, Vol. 682. New York: The New York Academy of Sciences.

Taylor, I., \& Taylor, M. M. (1995). Writing and literacy in Chinese, Korean and Japanese. Philadelphia, PA: John Benjamins Publishing Company.

Tzeng, O. J. T. (1982). On orthography and the relation between reading process and recognition. In H. S. R. Kao \& C. M. Cheng (Eds.), Psychological research on the Chinese language (pp. 85-101). Taipei: Wenhe.

Valtin, R. (1989). Dyslexia in the German language. In P. G. Aaron \& R. M. Joshi (Eds.), Reading and writing disorders in different orthographic systems (pp. 119-135). Dordrecht: Kluwer Academic Publishers.

Vellutino, F. R. (1987). Dyslexia. Scientific American, 256, 34-41. https://doi.org/10.1038/scientificamerican0387-34

Weigt, R. (1994). Lesen- und Schreibenlernen kann jeder!? Methodische Hilfen bei Lese-RechtschreibSchwaeche [Everyone can read and write!? Meth- odological aids in reading spelling weakness]. Neuwied, Kriftel, Berlin: Luchterhand.

Weiss, R. H. (1987). Grundintelligenztest Skala 2 - CFT 20 [Basic Intelligence Test Scale 2 - CFT 20]. Göttingen: Hogrefe.

Witruk, E. (1993). Memory deficits of dyslexic children. In P. Tallal, A. M. Galaburda, R. R. Llindas, \& C. von Euler (Eds.), Temporal information processing in the nervous system: Special reference to dyslexia and dysphasia. Annals of the New York Academy of Sciences (Vol. 682, pp. 430-435). New York: The New York Academy of Sciences.

Witruk, E., \& Rosendahl, W. (1999). Modalitaets-und Anforderungsspezifik von Arbeitsgedaechtnisleistungen bei Legasthenikern [Modality and demand specificity of working memory performances in dyslexics]. In Kongreßbericht der XXIII. Arbeits- und Fortbildungstagung der Deutschen Gesellschaft fuer Sprachheilpaedagogik e.V. Wuerzburg: edition von freisleben.

Witruk, E. (1999). Working memory performance in dyslexic children. In E. Witruk \& T. Lachmann (Eds.), Basic mechanisms of language and language disorders (pp. 20-21). Leipzig: Leipziger Universitaetsverlag.

Witruk, E., Ho, C. S., \& Schuster, U. (2002). Working memory in dyslexic children - How general is the deficit? In E. Witruk, A. D. Friederici, \& T. Lachmann (Eds.), Basic functions of language and language disorders (pp. 281-297). Boston, MA: Kluwer Academic Publishers.

Witruk, E. (2004). Specificity of perceptive and cognitive deficits in dyslexics from different language environments. In Proceedings of the International Conference on Cognitive Science. Allahabad, India.

Witruk E. (2005). Cultural dependency of perceptive and working memory deficits in dyslexics. In X. Feng \& H. Xinnian (Eds.), Cross-cultural studies in communication (pp. 22-29). Urumqui: Academic Publishers.

World Health Organization (1993). ICD-10. The international classification of diseases. Vol. 10: Classification of mental and behavioral disorders. Geneva: World Health Organization.

Zheng, G. (1998). The influence of culture, schooling and age on memory performance and competencies. A cross-cultural study on memory and metamemory development of Chinese and German children. Unpublished dissertation, University of Heidelberg.
Cantonese and German dyslexic children 